\title{
Non-Schlesinger Deformations of Ordinary Differential Equations with Rational Coefficients
}

\author{
A. V. Kitaev * \\ Steklov Mathematical Institute, Fontanka 27, St.Petersburg, 191011, Russia \\ and \\ Department of Pure Mathematics \\ University of Adelaide, Adelaide, SA 5005, Australia
}

June 15,2000

\begin{abstract}
We consider deformations of $2 \times 2$ and $3 \times 3$ matrix linear ODEs with rational coefficients with respect to singular points of Fuchsian type which don't satisfy the wellknown system of Schlesinger equations (or its natural generalization). Some general statements concerning reducibility of such deformations for $2 \times 2$ ODEs are proved. An explicit example of the general non-Schlesinger deformation of $2 \times 2$-matrix ODE of the Fuchsian type with 4 singular points is constructed and application of such deformations to the construction of special solutions of the corresponding Schlesinger systems is discussed. Some examples of isomonodromy and non-isomonodromy deformations of $3 \times 3$ matrix ODEs are considered. The latter arise as the compatibility conditions with linear ODEs with non-singlevalued coefficients.
\end{abstract}

2000 Mathematics Subject Classification: 34A20, 34E20, 33E30.

Short title: Non-Schlesinger Isomonodromy Deformations

*E-mail: kitaev@pdmi.ras.ru 


\section{Introduction}

It is well-known that isomonodromy deformations of the Fuchsian matrix ODE,

$$
\frac{d \Psi}{d \lambda}=\left(\frac{A_{0}}{\lambda}+\frac{A_{1}}{\lambda-1}+\frac{A_{t}}{\lambda-t}\right) \Psi
$$

where $A_{\infty}=A_{0}+A_{1}+A_{t}$ is normalized to be independent of $t$, are governed by the system of Schlesinger equations [1],

$$
\frac{d A_{0}}{d t}=\frac{1}{t}\left[A_{t}, A_{0}\right], \quad \frac{d A_{1}}{d t}=\frac{1}{t-1}\left[A_{t}, A_{1}\right], \quad \frac{d A_{t}}{d t}=\left[\frac{1}{t} A_{0}+\frac{1}{t-1} A_{1}, A_{t}\right] .
$$

This system is the compatibility condition of Eq. (1.1) with the following ODE,

$$
\frac{d \Psi}{d t}=-\frac{A_{t}}{\lambda-t} \Psi
$$

Throughout the paper we consider matrix ODEs with respect to independent variable $\lambda$ whose coefficients are rational functions of $\lambda$ with at least one Fuchsian singularity at $\lambda=t$. We call deformations of such ODEs Schlesinger (with respect to $t$ ) if these ODEs are compatible, with Eq. (1.3). This is a natural generalization of the notion of Schlesinger deformations of the Fuchsian ODEs. It is clear that the Schlesinger deformations are the simplest isomonodromy deformations. On the other hand, as it follows from the work by Malgrange [2] any isomonodromy deformation of the Fuchsian system could be described up to isomorphism by the Schlesinger deformations. The purpose of this work is to understand better what does "up to isomorphism" mean from the point of view of the theory of integrable systems and special functions of the isomonodromy type [3]. More precisely, the question is whether this isomorphism can be always constructed explicitly, or by other words can we obtain any new integrable systems or special functions by considering non-Schlesinger deformations of ODEs with rational coefficients?

The results presented in Sections 2 and 3 give a negative answer on this question in the case of the isomonodromy deformations: any integrable system, which describes nonSchlesinger isomonodromy deformations of any Fuchsian ODE (or weak non-Schlesinger (see Section 2) isomonodromy deformation for non-Fuchsian ODEs with rational coefficients) can be mapped explicitly by a proper Schlesinger transformation into some integrable system describing deformation of the Schlesinger type. This statement can be reformulated in the following way: there are no new transcendental functions defined via non-Schlesinger isomonodromy deformations of the Fuchsian ODEs. The sketch of this rather simple proof is given at the end of Section 3. It was communicated to me by A. A. Bolibruch for the case of the Fuchsian ODEs few weeks after the workshop where the results of this work were reported. Moreover, he pointed out to me his recent work [4], where he also studied non-Schlesinger deformations of the Fuchsian ODEs but looking at them from a different angle: the isomonodromy confluences of the Fuchsian singularities. However, A. A.Bolibruch did not study the reducibility of the ODEs subject to non-Schlesinger deformations which I address, in particular, in this work. The latter means further simplification of these ODEs. Moreover, such reducibility does not follow from the proof mentioned above and has a remarkable consequence for construction of the explicit solutions of the Painlevé and higher Painlevé equations, and (generalized) Garnier type systems. 
In Section 4 some simplest examples of non-isomonodromy deformations of $3 \times 3$ matrix ODEs, which are, of course, non-Schlesinger, are presented. Here for simplicity we consider non-Fuchsian type ODE since one of our tasks here is to show that nonisomonodromy deformations, which can be described via compatibility conditions (generalized Schlesinger equations), really exist. For the Fuchsian ODEs one can consider analogous examples. In our examples, presented in Section \&, appear only elementary and the Painlevé type functions. The latter non-evident fact is established (also after the workshop) by C. M. Cosgrove. However, to the best of my knowledge no general statements concerning reducibility of such deformations to the ones of the isomonodromy type are known. So that further interesting studies of such deformations are anticipated.

\section{Non-Schlesinger Isomonodromy Deformations of $2 \times 2$ Matrix ODEs}

We begin our study with the special case of Eq. (1.1) when $A_{k} \in \operatorname{sl}_{2}(\mathbb{C})$ for $k=0,1, t, \infty$. Let us suppose that $A_{\infty}=-\frac{\theta_{\infty}}{2} \sigma_{3}, \theta_{\infty} \neq 0$, which is in fact (excluding one exceptional solvable case) also a normalization rather than a restriction on $A_{k}$. In this case the system of Schlesinger equations (1.2) is equivalent to the sixth Painlevé equation [5].

Eq. (1.3) is a sufficient condition that monodromy matrices of a fundamental solution of Eq. (1.1) are independent of $t$. In general situation this condition is also necessary, but there are some special exceptional cases, which are discussed here. A possibility of such deformations resulted from the non-uniqueness of solubility of the inverse monodromy problem for some special monodromy matrices.

We begin with a simplest generalization of Eq. (1.3),

$$
\frac{d \Psi}{d t}=-\frac{A_{t}+\Lambda}{\lambda-t} \Psi
$$

Compatibility condition of Eqs. (1.1) and (2.1) reads,

$$
\begin{gathered}
{\left[\Lambda, A_{t}\right]=\Lambda,} \\
\frac{d A_{0}}{d t}=\frac{1}{t}\left[A_{t}+\Lambda, A_{0}\right], \quad \frac{d A_{1}}{d t}=\frac{1}{t-1}\left[A_{t}+\Lambda, A_{1}\right], \quad \frac{d A_{t}}{d t}=\left[\frac{1}{t} A_{0}+\frac{1}{t-1} A_{1}, A_{t}+\Lambda\right] .
\end{gathered}
$$

Eq. (2.2) implies: $\operatorname{tr} \Lambda=0, \Lambda^{2}=0$, and $\pm 1 / 2$ are the eigenvalues of $A_{t}$. Denote

$$
A_{t}=\left(\begin{array}{cc}
a_{t} & b_{t} \\
c_{t} & -a_{t}
\end{array}\right)
$$

then solution of Eq. (2.2) can be written as follows

$$
\Lambda=\mu\left(\begin{array}{cc}
-\left(a_{t}+1 / 2\right) b_{t} & -b_{t}^{2} \\
\left(a_{t}+1 / 2\right)^{2} & \left(a_{t}+1 / 2\right) b_{t}
\end{array}\right) \quad \text { for } \quad a_{t} \neq-1 / 2,
$$

and

$$
\Lambda=\mu\left(\begin{array}{cc}
c_{t} & 1 \\
-c_{t}^{2} & -c_{t}
\end{array}\right) \quad \text { for } \quad a_{t}=-1 / 2
$$

where $\mu=\mu(t)$ is an arbitrary function of $t$.

Consider now asymptotic expansion of a fundamental solution of Eq. (1.1) as $\lambda \rightarrow t$,

$$
\Psi=\sum_{k=0}^{\infty} \psi_{k}(\lambda-t)^{k}(\lambda-t)^{\sigma_{3} / 2}\left(\begin{array}{cc}
1 & \kappa \ln (\lambda-t) \\
0 & 1
\end{array}\right) C, \quad \operatorname{det} C=\operatorname{det} \psi_{0}=1,
$$


where the parameter $\kappa=0$ or 1 . One finds

$$
A_{t}=\frac{1}{2} \psi_{0} \sigma_{3} \psi_{0}^{-1}, \quad \Lambda=\left(C_{21}^{\prime} C_{22}-C_{22}^{\prime} C_{21}\right) \psi_{0}\left(\begin{array}{cc}
0 & 0 \\
1 & 0
\end{array}\right) \psi_{0}^{-1}
$$

where $C_{21}$ and $C_{22}$ are the corresponding matrix elements of $C$ and the primes denotes differentiation on $t$. The monodromy matrix of $\Psi$ corresponding to any loop, with only one singular point $\lambda=t$ inside, reads,

$$
M_{t}=-I-2 \pi i \kappa\left(\begin{array}{cc}
C_{21} C_{22} & C_{22}^{2} \\
-C_{21}^{2} & -C_{21} C_{22}
\end{array}\right)
$$

If $\kappa \neq 0$ then the isomonodromy condition, $\frac{d}{d t} M_{t}=0$, implies $C_{21}^{\prime} C_{22}-C_{22}^{\prime} C_{21}=0$ and therefore $\Lambda=0$. Thus, in the case of $2 \times 2$ matrices non-Schlesinger deformations of the type (2.1) exist if and only if $\kappa=0$. This condition can be written in terms of Eq. (1.1) as follows

$$
\operatorname{tr}\left(\left(\frac{1}{t} A_{0}+\frac{1}{t-1} A_{1}\right)\left(A_{t}+\frac{1}{2} \sigma_{3}\right)\right)=0
$$

Consider explicit construction of this deformation. Taking into account the integrals of the Schlesinger system (1.2),

$$
A_{t}=-A_{0}-A_{1}-\frac{\theta_{\infty}}{2} \sigma_{3}, \quad \operatorname{tr} A_{k}=0, \quad \operatorname{det} A_{k}=-\frac{\theta_{k}^{2}}{4}
$$

where $\theta_{t}=1$ and $\theta_{k}, k=0,1, \infty$ are the constants of integration considered as parameters; we see that the most general non-Schlesinger deformation of the type (2.1) depends on the function $\mu(t)$ (see Eqs. (2.4) and (2.5)) and five parameters (constants of integration of Eqs. (2.3)): $\theta_{0}, \theta_{1}, \theta_{\infty}$ and $c_{1}, c_{2}$. One of them can be introduced via the gauge transformation,

$$
A_{k} \longrightarrow c_{2}^{\sigma_{3}} A_{k} c_{2}^{-\sigma_{3}} \text {. }
$$

Due to the absence of the log-term in the expansion Eq. (2.6), the singularity at $\lambda=t$ in Eq. (1.1) is removable via a proper Schlesinger transformation. Let us describe this construction in detail. Consider the hypergeometric equation which can be written in the matrix form as follows,

$$
\frac{d \Phi}{d \lambda}=\left(\frac{A}{\lambda}-\frac{\frac{\theta_{\infty}+1}{2} \sigma_{3}+A}{\lambda-1}\right) \Phi, \quad A=\left(\begin{array}{cc}
-\frac{\theta_{0}^{2}-\theta_{1}^{2}+\left(\theta_{\infty}+1\right)^{2}}{4\left(\theta_{\infty}+1\right)} & \frac{\theta_{0}^{2}-\left(\theta_{\infty}+1-\theta_{1}\right)^{2}}{4\left(\theta_{\infty}+1\right)} \\
-\frac{\theta_{0}^{2}-\left(\theta_{\infty}+1+\theta_{1}\right)^{2}}{4\left(\theta_{\infty}+1\right)} & \frac{\theta_{0}^{2}-\theta_{1}^{2}+\left(\theta_{\infty}+1\right)^{2}}{4\left(\theta_{\infty}+1\right)}
\end{array}\right) .
$$

Note that $\operatorname{det} A=-\frac{\theta_{0}^{2}}{4}$ and $\operatorname{det}\left(\frac{\theta_{\infty}+1}{2} \sigma_{3}+A\right)=-\frac{\theta_{1}^{2}}{4}$. An explicit formula for the fundamental solution $\Phi=\Phi(\lambda)$ of this equation in terms of the Gauß hypergeometric functions can be found for example in [6]. In the following we denote as $\Phi$ any fundamental solution of Eq. (2.9) which is independent of $t$. A fundamental solution of the system (1.1), (1.3) which corresponds to the general (modulo gauge transformation (2.8)) solution of the system (2.1), (1.1) (for $\theta_{t}=1$ ) can be written as follows,

$$
\begin{aligned}
\Psi & =D^{-1} R^{-1}(\lambda, t) \Phi, \quad D=\left(\begin{array}{cc}
1 & 1 \\
0 & \kappa_{1}
\end{array}\right), \kappa_{1}=\frac{4 \theta_{\infty}\left(\theta_{\infty}+1\right)}{\left(\theta_{\infty}+1-\theta_{1}\right)^{2}-\theta_{0}^{2}} \\
R(\lambda, t) & =\left(\left(\begin{array}{cc}
0 & 0 \\
0 & 1
\end{array}\right) \sqrt{\lambda-t}+\left(\begin{array}{cc}
1 & 0 \\
c_{1}(t) & 0
\end{array}\right) \frac{1}{\sqrt{\lambda-t}}\right), \quad \operatorname{det} R(\lambda, t)=1,
\end{aligned}
$$


where $c_{1}(t)$ is an arbitrary function of $t$ and for both square roots in Eq. (2.11) should be chosen the same branch. The corresponding solution of the system (2.2), (2.3) reads:

$$
\begin{aligned}
& A_{0}=D^{-1} R^{-1}(0, t) A D R(0, t), \quad A_{1}=-D^{-1} R^{-1}(1, t)\left(\frac{\theta_{\infty}+1}{2} \sigma_{3}+A\right) D R(1, t), \\
& A_{t}=D^{-1}\left(\begin{array}{cc}
1 / 2 & 0 \\
r(t) & -1 / 2
\end{array}\right) D, \quad \Lambda=D^{-1}\left(\begin{array}{cc}
0 & 0 \\
\frac{d c_{1}(t)}{d t}-r(t) & 0
\end{array}\right) D
\end{aligned}
$$

where

$$
r(t)=\frac{\left(\theta_{0}^{2}-\left(\theta_{\infty}+1-\theta_{1}\right)^{2}\right) c_{1}^{2}(t)+\left(4 t\left(\theta_{\infty}+1\right)^{2}-2\left(\left(\theta_{\infty}+1\right)^{2}+\theta_{0}^{2}-\theta_{1}^{2}\right)\right) c_{1}(t)+\theta_{0}^{2}-\left(\theta_{\infty}+1+\theta_{1}\right)^{2}}{4 t(t-1)\left(\theta_{\infty}+1\right)} .
$$

The function $c_{1}(t)$ depends on the function $\mu(t)$ and the parameter $c_{1}$ which is introduced in the paragraph above Eq. (2.8). Actually, if the function $\mu(t)$ is given, as one of the coefficients in the system (2.2), (2.3), then

$$
\mu(t)=\frac{\kappa_{1}\left(c_{1}^{\prime}(t)-r(t)\right)}{\left(r(t)-\kappa_{1}\right)^{2}} .
$$

So that the function $c_{1}(t)$ is the general solution of the differential equation (2.14) and, therefore, depends on the constant of integration $c_{1}$.

At this stage it is worth to notice an application of the non-Schlesinger deformations to the construction of one-parameter families of solutions of the sixth Painlevé equation. As is mentioned above, the sixth Painlevé equation corresponds to the Schlesinger deformation (1.3) of Eq. (1.1), i.e., $\mu(t) \equiv 0 \Longrightarrow c_{1}^{\prime}(t)=r(t)$. The latter is nothing but the Riccati equation whose solution can be written in terms of the logarithmic derivative of the Gauß hypergeometric function. An explicit formula for the solution of the sixth Painlevé equation is than easy to get from the Eqs. (2.12) and a parametrization of the solution of the sixth Painlevé equation in terms of matrix elements of $A_{k}$ given in [5]. This solution corresponds to $\theta_{t}= \pm 1$.

It is important to notice that there is another construction of the one-parameter families of the solutions of the sixth Painlevé equation; it is based on on the "triangular reduction" of the system (1.1), (1.3) (the latter also leads to the hypergeometric functions, but for $\theta_{0}+\theta_{1}+\theta_{t}+\theta_{\infty}=0$, see, e.g., [3])). These two constructions give, modulo application of the Schlesinger transformations and fractional-linear transformations of $\lambda$, all one-parameter families of solutions of the sixth Painlevé equation. The fact that there are no other solutions of the sixth Painlevé equation follows from the work [7]. It is clear that application of the non-Schlesinger deformations to the construction of the special solutions of the Garnier systems and, so-called, higher Painlevé equations should "break the symmetry" with the construction of the special solutions based on the triangular reduction of the associated linear ODEs. The latter always leads to the linear ODEs for (generally multivariable) hypergeometric functions. Whilst the construction related with the non-Schlesinger deformations leads to the Riccati equations (for $2 \times 2$ matrix ODEs) which can be transformed to the linear ODEs of the second order whose coefficients are defined by solutions of the Garnier systems (Painlevé/higher Painlevé equations) which are junior members of the corresponding hierarchies.

The most general non-Schlesinger isomonodromy deformation of Eq. (1.1) are defined by the compatibility condition of Eq. (1.1) with the following ODE:

$$
\frac{\partial \Psi}{\partial t}=\left(-\frac{A_{t}+\Lambda_{1}^{t}}{(\lambda-t)}+\sum_{k=2}^{n_{t}} \frac{\Lambda_{k}^{t}}{(\lambda-t)^{k}}+\sum_{k=1}^{n_{1}} \frac{\Lambda_{k}^{1}}{(\lambda-1)^{k}}+\sum_{k=1}^{n_{0}} \frac{\Lambda_{k}^{0}}{\lambda^{k}}\right) \Psi,
$$


where $n_{t} \geq 1, n_{1} \geq 0$, and $n_{0} \geq 0$ are integers. The notation are chosen such that if $\Lambda_{n_{p}}^{p}=0$, then $\Lambda_{k}^{p}=0$ for all $k \leq n_{p}$ and if the upper limit of the sum is less than its lower limit, then the sum is absent. One proves, that $\operatorname{tr} \Lambda_{k}^{p}=0$ and $\left(\Lambda_{n_{p}}^{p}\right)^{2}=0$ for $p=t, 1,0$. Moreover,

$$
\Lambda_{n_{p}}^{p} \neq 0 \quad \Longrightarrow \quad A_{p}=\frac{n_{p}}{2} \psi_{0}^{p} \sigma_{3}\left(\psi_{0}^{p}\right)^{-1}, \quad \Lambda_{n_{p}}^{p}=\mu_{l_{p}}^{p}(t) \psi_{0}^{p}\left(\begin{array}{cc}
0 & 0 \\
1 & 0
\end{array}\right)\left(\psi_{0}^{p}\right)^{-1},
$$

the corresponding monodromy matrix at $\lambda=p$ is $M_{p}=(-1)^{n_{p}}$, this means that the function $\Psi$ has the following expansion at $\lambda=p$ :

$$
\Psi=\sum_{k=0}^{\infty} \psi_{k}(\lambda-p)^{k}(\lambda-p)^{\frac{n_{p}}{2} \sigma_{3}} C, \quad \operatorname{det} C=\operatorname{det} \psi_{0}=1 .
$$

It follows from this expansion that the singularity at $\lambda=p$ can be removed from Eqs. (1.1) and (2.15) via $n_{p}$ transformations of the type (2.10). In general, the non-Schlesinger isomonodromy deformation defined by Eq. 2.15) can be parametrized via $n_{p}+n_{1}+n_{0}$ arbitrary functions of $t$. More precisely: If among the "senior" matrices $\Lambda_{n_{k}}^{k}, k=0,1, t$, only one matrix $\Lambda_{n_{p}}^{p}$ is different from 0 , then via $n_{p}$ Schlesinger transformations of the form (2.10) and, possibly, (if $p \neq t$ ) by permutation of the points $0,1, t$; one transforms $\Psi$, the fundamental solution of the system (1.1), (2.15), to the solution of the hypergeometric equation (2.9) $\Phi$. If any two of the "senior" matrices $\Lambda_{n_{p}}^{p} \neq 0$ and $\Lambda_{n_{q}}^{q} \neq 0$, then $n_{p}+n_{q}$ Schlesinger transformations convert $\Psi$ to the the function $\Phi=(\lambda-r)^{-\left(\theta_{\infty}+1\right) \sigma_{3} / 2}$, where $r \neq p, q$ and $r \in\{0,1, t\}$, so that in particular $\theta_{r}=-\theta_{\infty}-1$. Finally, if all three matrices $\Lambda_{n_{k}}^{k} \neq 0, k=0,1, t$, then $\Psi$ can be presented as a multiplication of the $n_{t}+n_{0}+n_{1}$ Schlesinger transformations.

Since the consideration above is quite local this result can be generalized for $2 \times 2$ matrix ODEs

$$
\frac{d \Psi}{d \lambda}=A(\lambda) \Psi
$$

where $=A(\lambda) \in s l_{2}(\mathbb{C})$ is an arbitrary rational function of $\lambda$.

Definition. Let $t$ be a pole of $A(\lambda)$ of the first order with the residue $A_{t}$,

$$
A(\lambda)=B(\lambda)+\frac{A_{t}}{\lambda-t},
$$

where $B(\lambda)$ has no pole at $\lambda=t$ as a rational function of $\lambda$ and $A_{t}$ is independent of $\lambda$. If fundamental solution of Eq. (2.18) satisfies also Eq. (1.3), then we call any solution of the system representing compatibility condition of Eqs. (2.18), (2.19), and (1.3), namely,

$$
\frac{\partial B(\lambda)}{\partial t}=\left[\frac{B(\lambda)-B(t)}{\lambda-t}, A_{t}\right], \quad \frac{d A_{t}}{d t}=\left[B(t), A_{t}\right],
$$

the Schlesinger deformation of Eq. (2.18) with respect to the parameter $t$.

Remark 2.1 The fourth, fifth, and sixth Painlevé equations can be written as the Schlesinger deformations of $2 \times 2$ matrix linear ODEs.

Definition. Denote $t_{1}$ any other first order pole of the rational function $A(\lambda)$, in particular, it may coincide with $t$. We say, that isomonodromy deformation with respect to $t$ is $t_{1}$-non-Schlesinger, iff the rational function $R(\lambda)$ in the following equation,

$$
\frac{d \Psi}{d t}=\left(-\frac{A_{t}}{\lambda-t}+R(\lambda)\right) \Psi
$$


has a pole at $\lambda=t_{1}$.

Proposition 2.1 Any $t_{1}$-non-Schlesinger deformation of Eq. (2.18) with respect to $t$ can be transformed, by a finite number of the Schlesinger transformations of the type given by the first equation (2.10) where $t \rightarrow t_{1}$ and the matrix $D$ is independent of $\lambda$, to the $O D E$ of the form

$$
\frac{d \hat{\Phi}}{d \lambda}=\hat{A}(\lambda) \hat{\Phi}
$$

where the rational function $\hat{A}(\lambda) \in \operatorname{sl}_{2}(\mathbb{C})$ has no pole at $\lambda=t_{1}$ whilst its other poles and their orders coincide with those of the function $A(\lambda)$.

Moreover, if, additionally, the function $A(\lambda)$ is isomonodromic with respect to $t_{1}$, namely,

$$
\frac{d \Psi}{d t_{1}}=\left(-\frac{A_{t_{1}}}{\lambda-t_{1}}+R_{1}(\lambda)\right) \Psi
$$

and the set of poles $\left(\in \mathbb{C P}^{1}\right)$ of the rational function $R_{1}(\lambda)$ coincides with a subset $\left(\in \mathbb{C P}^{1}\right)$ of the first order poles of the function $A(\lambda)$, then, possibly by applying a finite number of additional Schlesinger transformations of the type described in the previous paragraph, one arrives at $E q$. (2.21), where the function $\hat{A}(\lambda)$ is independent of $t_{1}$ (note that if $t_{1}=t$, then $R(\lambda)=R_{1}(\lambda)$ has a pole at $\lambda=t$ ). The set of poles of $\hat{A}(\lambda)$ is a subset of the poles of $A(\lambda)$.

Remark 2.2 We call the non-Schlesinger deformations described in the second paragraph of Proposition 2.1 weak non-Schlesinger deformations. If one allows that among the poles of $R_{1}(\lambda)$ there are some non-Fuchsian singularities of Eq. (2.18), then such isomonodromy deformations could be called strong non-Schlesinger deformations. For the strong non-Schlesinger deformations the latter statement of Proposition 2.1, in general, is not true.

If we suppose that Eq. (2.18) suffers from some week non-Schlesinger deformation with respect to $t$ and $R(\lambda)$ (see Eq. (2.20)) has no pole at $\lambda=t$, then, by applying proper Schlesinger transformations, Eq. (2.18) can be simplified to Eq. (2.21) with the matrix $\hat{A}(\lambda)$ having less poles than $A(\lambda)$ and the Schlesinger $t$-dependence.

\section{Non-Schlesinger Isomonodromy Deformations of $3 \times 3$ matrix ODEs}

Whilst in the case when $2 \times 2$ matrix ODE (2.18) with the connection matrix 2.19) suffers from the weak non-Schlesinger isomonodromy deformations with respect to $t$, it is always reducible via the Schlesinger transformations to a simpler ODE with a less number of poles, this is not always the case for the ODEs of higher matrix dimension. For example, one can take a direct sum of two $2 \times 2$ equations of the type (1.1): coefficients of the first equation are deformed by the Schlesinger deformation (1.3), whilst coefficients of the second one suffer from a non-Schlesinger deformation of the type (2.1). Clearly, we have constructed a non-Schlesinger isomonodromy deformation of the $4 \times 4$ ODE with respect to $t$ which (in general situation) cannot be reduced via any Schlesinger transformation to any equation independent of $t$. However, it can be transformed (via an appropriate Schlesinger transformation) to the equation which deformation with respect 
to $t$ is of the Schlesinger type. So, the natural question is: are there any non-Schlesinger isomonodromy deformations of Eq. 2.18) which are not reducible via Schlesinger transformations to the Schlesinger deformations of some other ODE of the same type (2.18)?

Consider the simplest non-Schlesinger isomonodromy deformation of Eqs. (2.18), (2.19) of the type (2.1) for the case of $3 \times 3$ matrices. Eq. (2.2) now implies: $\operatorname{tr} \Lambda=0$, $\Lambda^{3}=0$. Using this one finds that the only possible solutions of Eq. (2.2) reads,

$$
\begin{aligned}
& \text { 1. } A_{t}=\psi_{0}\left(\begin{array}{ccc}
1 & 0 & 0 \\
0 & 0 & 0 \\
0 & 0 & -1
\end{array}\right) \psi_{0}^{-1}, \quad \Lambda=\psi_{0}\left(\begin{array}{ccc}
0 & 0 & 0 \\
\mu_{1}(t) & 0 & 0 \\
0 & \mu_{2}(t) & 0
\end{array}\right) \psi_{0}^{-1} \text {, } \\
& \text { 2. } A_{t}=\psi_{0}\left(\begin{array}{ccc}
1 / 3 & 0 & 0 \\
0 & 1 / 3 & 0 \\
0 & 0 & -2 / 3
\end{array}\right) \psi_{0}^{-1}, \quad \Lambda=\psi_{0}\left(\begin{array}{ccc}
0 & 0 & 0 \\
0 & 0 & 0 \\
\mu_{1}(t) & \mu_{2}(t) & 0
\end{array}\right) \psi_{0}^{-1} \text {, } \\
& \text { 3. } A_{t}=\psi_{0}\left(\begin{array}{ccc}
1 / 3 & 1 & 0 \\
0 & 1 / 3 & 0 \\
0 & 0 & -2 / 3
\end{array}\right) \psi_{0}^{-1}, \quad \Lambda=\psi_{0}\left(\begin{array}{ccc}
0 & 0 & 0 \\
0 & 0 & 0 \\
0 & \mu_{2}(t) & 0
\end{array}\right) \psi_{0}^{-1} \text {, } \\
& \text { 4. } A_{t}=\psi_{0}\left(\begin{array}{ccc}
2 / 3 & 0 & 0 \\
0 & -1 / 3 & 0 \\
0 & 0 & -1 / 3
\end{array}\right) \psi_{0}^{-1}, \quad \Lambda=\psi_{0}\left(\begin{array}{ccc}
0 & 0 & 0 \\
\mu_{1}(t) & 0 & 0 \\
\mu_{2}(t) & 0 & 0
\end{array}\right) \psi_{0}^{-1} \text {, } \\
& \text { 5. } A_{t}=\psi_{0}\left(\begin{array}{ccc}
2 / 3 & 0 & 0 \\
0 & -1 / 3 & 1 \\
0 & 0 & -1 / 3
\end{array}\right) \psi_{0}^{-1}, \quad \Lambda=\psi_{0}\left(\begin{array}{ccc}
0 & 0 & 0 \\
\mu_{1}(t) & 0 & 0 \\
0 & 0 & 0
\end{array}\right) \psi_{0}^{-1} \text {. }
\end{aligned}
$$

The matrix $\psi_{0}$ is a function of $t$ with $\operatorname{det} \psi_{0}=1, \mu_{1}(t)$ and $\mu_{2}(t)$ are some functions of $t$. Actually, from the system (2.3) follows that for any choice of the functions $\mu_{k}(t)$ there is a solution of the system (2.3). This means that general solution in each case depends on two (the cases $1,2,4$ ) or one (the cases 3,5 ) arbitrary functions of $t$. The functions $\mu_{1}(t)$ and $\mu_{2}(t)$ may also depend on some other pole parameters (if any).

Consider the first case. Any fundamental solution at $\lambda=t$ has the following asymptotic expansion,

$$
\begin{aligned}
& \Psi \underset{\lambda \rightarrow t}{=} \sum_{k=0}^{\infty} \psi_{k}(\lambda-t)^{k}\left(\begin{array}{ccc}
\lambda-t & 0 & 0 \\
0 & 1 & 0 \\
0 & 0 & 1 /(\lambda-t)
\end{array}\right)(\lambda-t)^{\Xi} C, \\
& \Xi=\left(\begin{array}{ccc}
0 & \kappa_{2} & \kappa_{1} \\
0 & 0 & \kappa_{3} \\
0 & 0 & 0
\end{array}\right), \quad \operatorname{det} C=\operatorname{det} \psi_{0}=1,
\end{aligned}
$$

where $\kappa_{l}, l=1,2,3$ are parameters independent of $t$. Denote

$$
Q=C^{\prime}(t) C^{-1}(t), \quad \Rightarrow \quad \operatorname{tr} Q=0 .
$$

Now using expansion (3.6) one proves that

$$
\begin{aligned}
\frac{d \Psi}{d t} & =\left(\frac{\Lambda_{2}}{(\lambda-t)^{2}}-\frac{A_{t}+\Lambda}{\lambda-t}\right) \Psi, \\
\Lambda_{2} & =\psi_{0}\left(\begin{array}{ccc}
0 & 0 & 0 \\
0 & 0 & 0 \\
Q_{31} & 0 & 0
\end{array}\right) \psi_{0}^{-1}, \quad \Lambda=-\psi_{0}\left(\begin{array}{ccc}
0 & 0 & 0 \\
Q_{21} & 0 & 0 \\
0 & Q_{32} & 0
\end{array}\right) \psi_{0}^{-1},
\end{aligned}
$$


where $Q_{i k}$ are matrix elements of $Q$ and $A_{t}$ is given by Eq. (3.1). In fact, Eq. (2.1) is a special case of Eq. (3.8) corresponding to the case $Q_{31}=0$. The monodromy matrix of the fundamental solution with expansion (3.6) corresponding to a small loop around $\lambda=t$ reads,

$$
M_{t}=C^{-1} e^{2 \pi i \Xi} C
$$

So that the isomonodromy condition, $\frac{d}{d t} M_{t}=0$ is equivalent to

$$
\left[Q, e^{2 \pi i \Xi}\right]=0 .
$$

The result of the analysis of Eq. (3.10) can be formulated as the following

Proposition 3.1 In generic situation, i.e., $\nu(t)=Q_{31} \neq 0$ or $\nu(t)=Q_{31}=0$ but $\mu_{1}(t) \mu_{2}(t)=Q_{21} Q_{32} \neq 0$, the isomonodromy condition (3.10) implies $\Xi=0$; therefore, there is a Schlesinger transformation which transfer Eq. (2.18) with the Fuchsian singularity at $\lambda=t$ into the equation of the same type but without this singularity.

If $\nu(t)=0, \mu_{2}(t)=0$ but $\mu_{1}(t) \neq 0$, then $\kappa_{2}=0$. If $\nu(t)=0, \mu_{1}(t)=0$ but $\mu_{2}(t) \neq 0$, then $\kappa_{3}=0$.

Remark 3.1 In each of the two last cases of Proposition 3.1 there are some further necessary conditions on $Q$, following from Eq. (3.10), which allow to have $\Xi \neq 0$. The question is whether these conditions are sufficient, namely, can one construct a nonSchlesinger isomonodromy deformation of the type (2.1) for some ODE of the form (2.18) such that Eq. (3.10) is satisfied and the matrix $\Xi \neq 0$, is opened.

The analysis of the cases 2 and 4 (see Eq. (3.2) and (3.4)) essentially repeats the previous one. Some minor modifications, say, for the case 2 are as follows. Matrix $\Lambda_{2}=0$, $\mu_{1}(t)=Q_{31}$, and $\mu_{2}(t)=Q_{32}$. Instead of expansion (3.6) we have

$$
\Psi \underset{\lambda \rightarrow t}{=} \sum_{k=0}^{\infty} \psi_{k}(\lambda-t)^{k}\left(\begin{array}{ccc}
(\lambda-t)^{\frac{1}{3}} & 0 & 0 \\
0 & (\lambda-t)^{\frac{1}{3}} & 0 \\
0 & 0 & (\lambda-t)^{-\frac{2}{3}}
\end{array}\right)(\lambda-t)^{\Xi} C,
$$

where the matrices $\Xi$ and $C$ have the same properties as those in (3.7). The monodromy matrix around $\lambda=t$ reads $M_{t}=e^{2 \pi i / 3} C^{-1} e^{\Xi} C$, therefore isomonodromy condition again reduces to Eq. (3.10). So that the part of Proposition 3.1 for $\nu(t)=0$ and Remark 3.1 apply also for the cases 2 and 4 without any modifications.

Minor modifications are required also in the remaining cases 3 and 5 (see Eq. (3.3) and (3.5) ). In the case 3 expansion of the function $\Psi$ at $\lambda=t$ is just a special case of the expansion corresponding to the case 2 (3.11) in which $\kappa_{1}=0$ and $\kappa_{2}=1$ see [8]. The non-Schlesinger condition $\mu_{2}(t)=Q_{32} \neq 0$ implies $\kappa_{3}=0$. Similarly, the non-Schlesinger condition in the case 5, i.e., $\mu_{1}(t)=0$, leads to the condition $\kappa_{1}=0, \kappa_{2}=0$, and $\kappa_{3}=1$. In these cases the log-terms in the corresponding asymptotic expansions remain, so that these singularities definitely cannot be removed via Schlesinger transformations.

Nevertheless, in the cases 3 and 5 one can transform the non-Schlesinger deformations (2.1) to the Schlesinger form (1.1) via the Schlesinger transformations. Although, in this case one cannot reduce the number of poles in the transformed version of Eq. (2.18) like it can be done in the other cases considered above. Actually, the idea of this proof is quite

\footnotetext{
${ }^{1}$ For the Fuchsian systems the idea of the proof was communicated to me by A. A. Bolibruch.
} 
simple and works for general weak non-Schlesinger deformations of Eq. (2.18) in $n \times n$ matrices. However, to avoid long introduction of the notation, I'll explain it by using the redundant notation, which are already introduced. Suppose that Eq. (2.20) defines some weak non-Schlesinger deformation of Eq. (2.18). Denote $\left\{t_{1}, \ldots, t_{m}\right\}$ the set of poles of the function $R(\lambda)$. One of them may coincide with $t$ and simultaneously they belong to the set of the first order poles of Eq. (2.18). Note, that $t_{1}, \ldots, t_{m}$ are not supposed to be the first order poles of $R(\lambda)$. Denote, $C_{1}(t), \ldots, C_{m}(t)$ and $\Xi_{1}, \ldots, \Xi_{m}$ the matrices defining pole expansions (the matrices like $C(t)$ and $\Xi$ in Eqs. (3.6)) and (3.11) of the solution $\Psi$ at the points $t_{1}, \ldots, t_{m}$, respectively. The matrices $C_{k}(t)$ may also depend on some other parameters, in particular, on $t_{k}$, whilst the upper nilpotent matrices $\Xi_{k}$ are chosen to be constants. The key observation is that the matrices $C_{k}(t) C_{k}^{-1}\left(t_{0}\right)$, where $t_{0}$ $\notin\left\{t_{1}, \ldots, t_{m}\right\}$ is any point chosen such that all matrices $C_{k}\left(t_{0}\right)$ are invertible, commute with $\Xi_{k}$. This fact follows from the isomonodromy condition: for any monodromy matrix $M_{k}(t)=M_{k}\left(t_{0}\right)$. The inverse monodromy problem at $t=t_{0}$ is solvable, since it is supposed that the matrices $C_{k}\left(t_{0}\right)$ exist, therefore, we can construct the Schlesinger deformation with the same monodromy data as the ones for the function $\Psi$, but with $C_{k}(t) \rightarrow C_{k}\left(t_{0}\right)$. This fact follows from the solubility of the Cauchy problem at $t=t_{0}$ for the (generalized) Schlesinger system for the coefficients of Eq. (2.18). Now, denote $\Psi_{S}$ the solution corresponding to the Schlesinger deformations of Eq. (2.18). Consider analytic properties of $\Psi \Psi_{S}^{-1}$ as the function of $\lambda \in \mathcal{C} \mathcal{P}^{1}$ and prove (Liouville theorem) that it is a rational function of $\lambda$ (here the commutativity $C_{k}(t) C_{k}^{-1}\left(t_{0}\right)$ with $\Xi_{k}$ is used). So, $\Psi$ and $\Psi_{S}$ are related by some Schlesinger transformation.

It is an interesting problem to classify all cases when weakly non - Schlesinger Eq. (2.18) in $n \times n$ matrices can be reduced to a simplified equation of the same type which is either independent or "Schlesinger" with respect to the corresponding parameter(s).

\section{Some Examples of non-Isomonodromy Deformations of $3 \times 3$ matrix ODEs}

It is easy to see that any function $\Psi$ which solves Eq. (2.18) with r.-h.s. given by Eq. 2.19), where $B(\lambda)$ is a rational function of $\lambda$ holomorphic at $\lambda=t$, and depends isomonodromicaly on the parameter $t$, satisfies also equation

$$
\frac{d \Psi}{d t}=U(\lambda, t) \Psi
$$

where $U(\lambda, t)$ is a rational function of $\lambda$. At the same time there are some deformations of Eq. (2.18) which defined by Eq. (4.1) with a non-rational matrix $U(\lambda, t)$, so that these deformations are non-isomonodromy. One interesting example of the non-isomonodromy deformations is considered in [9]. In that example, non-rational contribution to the matrix $U(\lambda, t)$ is proportional to the identity matrix and therefore reducible to isomonodromy deformations of the Schlesinger type via $\lambda$ independent gauge transformations. Here, we consider some simplest examples of the non-isomonodromy deformations for the following $3 \times 3$ matrix ODE,

$$
\frac{d \Psi}{d \lambda}=\left(\frac{A_{0}}{\lambda}+\frac{A}{\lambda-t}+\left(\begin{array}{ccc}
\theta & 0 & 0 \\
0 & \theta & 0 \\
0 & 0 & -2 \theta
\end{array}\right)\right) \Psi,
$$


where $\theta$ is a parameter. Non-isomonodromy deformations considered below are also reducible to the isomonodromy ones, however, these reductions less straightforward as in the example given in 9 .

The first deformation is defined by the following ODE,

$$
\frac{d \Psi}{d t}=\left(-\frac{A}{\lambda-t}+P \ln (\lambda-t)\right) \Psi, \quad P=\left(\begin{array}{lll}
0 & 1 & 0 \\
1 & 0 & 0 \\
0 & 0 & 1
\end{array}\right)
$$

Denote

$$
U=A_{0}+A, \quad \Theta=\operatorname{diag}\{\theta, \theta,-2 \theta\}
$$

then compatibility condition of Eqs. (4.2) and (4.3) reads,

$$
\begin{aligned}
& P A_{0}=A_{0} P, \quad, P U=U P, \quad P \Theta=\Theta P, \\
& A_{0}^{\prime}=\frac{1}{t}\left[U, A_{0}\right], \quad U^{\prime}=P+\left[\Theta, U-A_{0}\right] .
\end{aligned}
$$

Solution of the system (4.4) and (4.5) can be written as follows,

$$
\begin{aligned}
A_{0} & =\left(\begin{array}{ccc}
a_{1}(t) & a_{1}(t)+\theta_{1} & a_{3}(t) \\
a_{1}(t)+\theta_{1} & a_{1}(t) & a_{3}(t) \\
a_{2}(t) & a_{2}(t) & -2 a_{1}(t)-\theta_{2}
\end{array}\right), \\
U & =\left(\begin{array}{ccc}
\theta_{4} & t+\theta_{5} & u_{3}(t) \\
t+\theta_{5} & \theta_{4} & u_{3}(t) \\
u_{2}(t) & u_{2}(t) & t+\theta_{0}+\theta_{4}+\theta_{5}
\end{array}\right)
\end{aligned}
$$

where $\theta_{k}, k=0, \ldots, 5$ are parameters and

$$
\begin{gathered}
4 a_{1}(t)+\theta_{1}+\theta_{2}=\sqrt{\theta_{3}^{2}-8 a_{2}(t) a_{3}(t)}, \quad a_{3}(t)=-\frac{u^{\prime}(\tau)}{2 \sqrt{2}} e^{\tau}, \quad a_{2}(t)=\frac{v^{\prime}(\tau)}{2 \sqrt{2}} e^{-\tau}, \\
u_{3}(t)=\frac{u(\tau)}{2 \sqrt{2}} e^{\tau}, \quad u_{2}(t)=\frac{v(\tau)}{2 \sqrt{2}} e^{-\tau}, \quad \tau=3 \theta t .
\end{gathered}
$$

The functions $u=u(\tau)$ and $v=v(\tau)$ satisfy the following system,

$$
\begin{aligned}
\tau u^{\prime \prime} & =u \sqrt{\theta_{3}^{2}+u^{\prime} v^{\prime}}-\left(\theta_{0}+\tau\right) u^{\prime}, \\
\tau v^{\prime \prime} & =v \sqrt{\theta_{3}^{2}+u^{\prime} v^{\prime}}+\left(\theta_{0}+\tau\right) v^{\prime} .
\end{aligned}
$$

This system can be reduced to one ODE of the second order. To prove it let us define auxiliary variables,

$$
f=u v, \quad g=\sqrt{\theta_{3}^{2}+u^{\prime} v^{\prime}}, \quad h=u^{\prime} v-v^{\prime} u .
$$

One finds the following equations,

$$
\begin{aligned}
& f^{\prime}=2 \tau g^{\prime}, \quad h^{\prime}=-2\left(\theta_{0}+\tau\right) g^{\prime}, \quad \tau h^{\prime}=-\left(\theta_{0}+\tau\right) f^{\prime}, \\
& f^{\prime 2}=h^{2}+4\left(g^{2}-\theta_{3}^{2}\right) f, \quad 2 \theta_{0} g+f+h=\kappa,
\end{aligned}
$$

where $\kappa$ is the constant of integration. By excluding the function $h$ we arrive to the following system of ODEs

$$
\begin{aligned}
& \tau\left(\left(\tau g^{\prime}\right)^{\prime}-g^{2}+\theta_{3}^{2}\right)+\frac{1}{2}\left(\theta_{0}+\tau\right)\left(\kappa-2 \theta_{0} g\right)=\frac{1}{2}\left(2 g+\theta_{0}+\tau\right) f, \\
& \left(\tau g^{\prime}\right)^{2}=\frac{1}{4}\left(\kappa-2 \theta_{0} g-f\right)^{2}+\left(g^{2}-\theta_{3}^{2}\right) f .
\end{aligned}
$$


Finding $f$ from the first equation and substituting it into the second one, we get an ODE for the function $g$ which is quadratic with respect to the second derivative. This equation can be solved in terms of the fifth Painlevé transcendent: the following derivation of this fact is due to C. M. Cosgrove.

First, observe that $f$ can be eliminated to give the following second-order seconddegree differential equation for $g$ :

$$
\begin{aligned}
& \left(\tau^{2} g^{\prime \prime}+\tau g^{\prime}+2 g^{3}+3 \theta_{0} g^{2}-\left(\kappa+2 \theta_{3}^{2}\right) g-\theta_{0} \theta_{3}^{2}\right)^{2} \\
= & \left(2 g+\tau+\theta_{0}\right)^{2}\left(\tau^{2}\left(g^{\prime}\right)^{2}+\left(g^{2}-\theta_{3}^{2}\right)\left(g^{2}+2 \theta_{0} g-\kappa-\theta_{3}^{2}\right)\right) .
\end{aligned}
$$

Equations gauge-equivalent to (4.10) were known to Chazy [11 and Bureau 12]. The singular integral is immediately apparent: if the last factor on the right-hand side is set to zero, then the left-hand side also vanishes and $g(\tau)$ becomes an elliptic function of the variable $\log \tau$.

To get the general integral, first observe that $g^{\prime \prime}$ can be eliminated between the first equation in (4.9) and the derivative of the second. The result can be factorised as

$$
\left(f^{\prime}-2 \tau g^{\prime}\right)\left(f+2 g^{2}+2 \theta_{0} g-\kappa-2 \theta_{3}^{2}\right)=0 .
$$

The second factor yields the singular integral again. The first factor, which will lead to the general integral, vanishes when we express $f$ and $g$ in terms of an auxiliary variable $H(\tau)$ according to

$$
f=-4\left(\tau H^{\prime}-H\right)-\theta_{0}^{2}, \quad g=-2 H^{\prime}-\frac{1}{2} \theta_{0} .
$$

When $f$ and $g$ are eliminated in favour of $H$, we obtain the following second-order second-degree equation:

$$
\tau^{2}\left(H^{\prime \prime}\right)^{2}=-4\left(H^{\prime}\right)^{2}\left(\tau H^{\prime}-H\right)+A_{1}\left(\tau H^{\prime}-H\right)^{2}+A_{2}\left(\tau H^{\prime}-H\right)+A_{3} H^{\prime}+A_{4},
$$

where

$$
\begin{aligned}
& A_{1}=1, \quad A_{2}=\frac{1}{4}\left(3 \theta_{0}^{2}+4 \theta_{3}^{2}+2 \kappa\right), \quad A_{3}=\frac{1}{2} \theta_{0}\left(\theta_{0}^{2}+\kappa\right), \\
& A_{4}=\frac{1}{16}\left(\left(\theta_{0}^{2}+\kappa\right)\left(3 \theta_{0}^{2}+\kappa\right)+4 \theta_{0}^{2} \theta_{3}^{2}\right) .
\end{aligned}
$$

Equation (4.13) or a gauge-equivalent version appears in several references, including [11, 12, 10. We have presented it in the standard form of equation SD-I.b in [10]. Its general solution is

$$
\begin{aligned}
H & =\frac{1}{4 w}\left(\frac{\tau w^{\prime}}{w-1}-w\right)^{2}-\frac{1}{4}\left(\theta_{0}^{2}+\theta_{3}^{2}+\kappa\right)(w-1) \\
& +\frac{\theta_{3}^{2}(w-1)}{4 w}+\frac{\theta_{0} \tau(w+1)}{4(w-1)}-\frac{\tau^{2} w}{4(w-1)^{2}},
\end{aligned}
$$

where the variable $w(\tau)$ is any solution of the Painlevé-V equation,

$$
w^{\prime \prime}=\left(\frac{1}{2 w}+\frac{1}{w-1}\right)\left(w^{\prime}\right)^{2}-\frac{1}{\tau} w^{\prime}+\frac{(w-1)^{2}}{\tau^{2}}\left(\alpha w+\frac{\beta}{w}\right)+\frac{\gamma w}{\tau}+\frac{\delta w(w+1)}{w-1}
$$


having parameters,

$\alpha=\frac{1}{2}(1+\sigma)^{2}, \quad$ where $\quad \sigma:= \pm \sqrt{\theta_{0}^{2}+\theta_{3}^{2}+\kappa}, \quad \beta=-\frac{1}{2} \theta_{3}^{2}, \quad \gamma=\theta_{0}, \quad \delta=-\frac{1}{2}$.

The Schlesinger and Lukashevich transformations admitted by the Painlevé-V equation induce corresponding symmetries in the $f, g$-system (4.9). One of these, which is easily found directly, is the involution,

$$
\begin{aligned}
f & \rightarrow f-2 \theta_{3}\left(\theta_{3}+\sigma\right)-\kappa, \quad g \rightarrow g+\frac{1}{2}\left(\theta_{0}-\theta_{3}-\sigma\right), \quad \sigma \rightarrow \frac{1}{2}\left(\theta_{0}-\theta_{3}+\sigma\right), \\
\theta_{0} & \rightarrow \theta_{3}+\sigma, \quad \theta_{3} \rightarrow \frac{1}{2}\left(\theta_{0}+\theta_{3}-\sigma\right), \quad \kappa \rightarrow\left(\theta_{0}-2 \theta_{3}\right) \sigma-\theta_{0}^{2}-\theta_{0} \theta_{3}-2 \theta_{3}^{2}-\kappa .
\end{aligned}
$$

As is well known, there are several classes of Painlevé-V equations that can be transformed into the Painlevé-III equation,

$$
u^{\prime \prime}=\frac{\left(u^{\prime}\right)^{2}}{u}-\frac{u^{\prime}}{\tau}+\tilde{\alpha} u^{3}+\frac{\tilde{\beta}}{\tau} u^{2}+\frac{\tilde{\gamma}}{\tau}+\frac{\tilde{\delta}}{u} .
$$

The case $\theta_{0}=\kappa=0$, with $\theta_{3}$ arbitrary, is one such example. In terms of an auxiliary variable $p(\tau)$, we have

$$
f=p^{2}, \quad g=\tau p^{-1}\left(p^{\prime \prime}-\frac{1}{4} p\right)
$$

where $p$ satisfies the second-order second-degree equation,

$$
\tau^{2}\left(p^{\prime \prime}-\frac{p}{4}\right)^{2}=p^{2}\left(\left(p^{\prime}\right)^{2}-\frac{p^{2}}{4}+\theta_{3}^{2}\right),
$$

Equation (4.18) is a special case of an equation appearing in [11, 12, 10]. Its solution is given by

$$
p=\tau u^{-1}\left(u^{\prime}+u^{2}-\frac{1}{16}\right)
$$

where $u(\tau)$ is any solution of the Painlevé-III equation (4.16) with parameters,

$$
\tilde{\alpha}=1, \quad \tilde{\beta}=-\left(1 \pm 2 \theta_{3}\right), \quad \tilde{\gamma}=\frac{1}{16}\left(1 \pm 2 \theta_{3}\right), \quad \tilde{\delta}=-\frac{1}{256} .
$$

The involution $u \rightarrow 1 /(16 u)$ induces the reflection $p \rightarrow-p$, which leaves $f$ and $g$ invariant. The above involution admitted by $f$ and $g$ induces a similar Painlevé-III solution of the $f, g$-system having $\kappa=-\theta_{0}^{2}$ and $\theta_{3}=0$.

Of course, we can choose more general form of the matrix $P$ in these examples, in particular it is clear that everything go through for $P \longrightarrow \mu(t) P$ where $\mu(t)$ is an arbitrary function. This will lead to a generalization of Eq. (4.9) which contains the function $\mu(t)$. However, most likely the latter equation will be again equivalent to the fifth Painlevé equation, since as is shown in the work [10] the class of equations quadratic with respect to the second derivatives and solvable in terms of the Painlevé transcendents contains equations with the coefficients depending on some arbitrary functions which are not removable by simple scaling transformations.

An explanation of the appearance of the Painlevé transcendents in the description of the non-isomonodromy deformations is that these deformations preserve some subset of the 
monodromy data of Eq. (4.2). More precisely, the following transformation, $\Psi=Q \tilde{\Psi}$ where the matrix

$$
Q=\left(\begin{array}{ccc}
0 & 1 & 1 \\
0 & 1 & -1 \\
1 & 0 & 0
\end{array}\right)
$$

diagonalizes $P, Q^{-1} P Q=(1,1,-1)$; transforms system (4.2), (4.3) to the block form,

$$
\left(\begin{array}{lll}
* & * & 0 \\
* & * & 0 \\
0 & 0 & *
\end{array}\right)
$$

Therefore, denoting $\hat{A}$ and $\hat{A}_{0} 2 \times 2$ minors with non-vanishing elements of the matrices $Q^{-1} A Q$ and $Q^{-1} A_{0} Q$ correspondingly, one finds that vector, $\hat{\Psi}$, formed with the first two components of the vector $\tilde{\Psi}$, solves the following system of $2 \times 2$ ODEs,

$$
\frac{d \hat{\Psi}}{d \lambda}=\left(\left(\begin{array}{cc}
-2 & 0 \\
0 & 1
\end{array}\right)+\frac{\hat{A}_{0}}{\lambda}+\frac{\hat{A}}{\lambda-t}\right) \hat{\Psi}, \quad \frac{d \hat{\Psi}}{d t}=-\frac{\hat{A}}{\lambda-t} \hat{\Psi} .
$$

As is well known from [5] the monodromy data of fundumental solutions of system 4.20] are independent of $t$ and correspoding isomonodromy deformations of its coefficients are governed by the fifth Painlevé equation. This is another derivation of the relation of the system (4.9) with the fifth Painlevé equation.

Another deformation of Eq. (4.2) can be defined as follows,

$$
\frac{d \Psi}{d t}=\left(-\frac{A}{\lambda-t}+P_{1}(\lambda-t)^{\alpha}\right) \Psi, \quad P_{1}=\left(\begin{array}{ccc}
0 & 1 & 0 \\
0 & 0 & 0 \\
0 & 0 & 0
\end{array}\right) .
$$

The compatibility condition of (4.21) with (4.2) reads

$$
\begin{aligned}
& {\left[\Theta, P_{1}\right]=\left[A_{0}, P_{1}\right]=0, } {\left[A_{1}, P_{1}\right]=\alpha P_{1}, } \\
& A_{0}^{\prime}=\frac{1}{t}\left[A_{1}, A_{0}\right], \quad A_{1}^{\prime}=\frac{1}{t}\left[A_{0}, A_{1}\right]+\left[\Theta, A_{1}\right] .
\end{aligned}
$$

In the case of $3 \times 3$ matrices the system (4.22), (4.23) reduces to the equation for the confluent hypergeometyric functions, for higher matrix dimensions it is a system of nonlinear ODEs.

One can consider more complicated than (4.3) and (4.21) non-isomonodromy deformations of Eq. (4.2) by adding into the r.-h.s.'s of these equations some further terms (like $\log ^{2}$, etc.). I believe that such non-isomonodromy deformations requires further study. In particular, a combination of the non-Schlesinger terms, the terms considered in this and the previous sections, could possibly lead to non-Painlevé type equations.

Acknowledgment I am grateful to A. A. Bolibruch who called my attention (after this work has been done) to his work [4] and for subsequent valuable discussions, and Nalini Joshi who pointed me out the work [9]. I am also indebt to C. M. Cosgrove for his help in the identification of equations (4.8) and (4.18). I would like to thank A. P. Fordy and V. B. Kuznetsov, the organizers of the Workshop Mathematical Methods in Regular Dynamics, for the invitation and partial financial support. My participation was also partially supported by Russian Foundation for Basic Research and Australian Research Council. 


\section{References}

[1] L. Schlesinger, Über eine Klasse von differentialsystemen beliebiger Ordnung mit festen Kritischen Punkten, J. für Math. 141, 96-145 (1912).

[2] B. Malgrange, Sur les deformations isomonodromiques I. Singularités régulières, in: Mathématique et Physique, Séminaire de l'Ecole Normale Supérieure 1979-1982, Progress in Mathematics 37, Birkhäser, Boston, 401-426, (1983).

[3] A. V. Kitaev, Special Functions of the Isomonodromy Type, Acta Appl. Math. 64, no. 1, 1-32 (2000).

[4] A. A. Bolibruch, On Isomonodromic Confluences of Fuchsian Singularities, Proceedings of the Steklov Institute of Mathematics, 221, 117-132 (1998).

[5] M. Jimbo and T. Miwa, Monodromy preserving deformation of linear ordinary differential equations with rational coefficients II, Physica 2D, 407-448 (1981).

[6] M. Jimbo, Monodromy problem and the boundary condition for some Painlevé equations, Publ. RIMS, Kyoto Univ., 18, 1137-1161 (1982).

[7] Huminiko Watanabe, Birational canonical transformations and classical solutions of the sixth Painlevé equation, Ann. Scuola Norm. Sup. Pisa Cl. Sci.(4) (1998), 27, no. 3-4, 379-425 (1999).

[8] F. R. Gantmacher, Applications of the Theory of Matrices, Interscience Publishers, Inc., New York, 1959.

[9] S. Chakravarty and M. J. Ablowitz, Integrability, monodromy evolving deformations, and self-dual Bianchi IX systems. Phys. Rev. Lett., 76, no. 6, 857-860 (1996).

[10] C. M. Cosgrove and G. Scoufis, Painlevé classification of a class of differential equations of the second order and second degree, Studies in Applied Mathematics 88, 25-87 (1993).

[11] J. Chazy, Sur les équations différentielles du second ordre à points critiques fixes, Comptes Rendus de l'Académie des Sciences, Paris 148, 1381-1384 (1909).

[12] F. Bureau, Équations différentielles du second ordre en $Y$ et du second degré en $\ddot{Y}$ dont l'intégrale générale est à points critiques fixes, Annali di Matematica 91, 163-281 (1972). 